\title{
Analysis of the influence of the current drawn by the appliance on the close magnetic field
}

\author{
Silviu Ursache ${ }^{1}$, Eduard Lunca ${ }^{1}$, Alexandru Salceanu ${ }^{1}$, Ionel Pavel ${ }^{1}$ \\ 1 "Gheorghe Asachi" Technical University of lasi, Prof. Dr. Doc. D. Mangeron Street, 700050 laşi, Romania
}

\section{ABSTRACT}

The development of technology and the corresponding increase in living standards imply the generalized use of the most diversified and sophisticated equipment, a distinct, significant category being household appliances. The frequent presence of these devices close to a person's heart, eyes, or brain naturally gives rise to concerns about the level of the associated (low-frequency) magnetic fields. The main objective of our paper is to study the possible correspondence between the low-frequency magnetic field generated by some household appliances and their current consumption. The proposed measurement system consists of the hand-held triple axis 480826 EMF Tester manufactured by Extech Instruments and the $80 \mathrm{i}-400$ Fluke clamp meter. The performed measurement methodology takes into account the operating principles of the appliance. We perform measurements for a large selection of devices (47). The measuring point for the magnetic field is set at $30 \mathrm{~cm}$ from the source. Finally, by applying the Biot-Savart law to the previously determined drawn current, we calculate the magnetic field. We also compare the corresponding, directly measured values and analyze them.

\section{Section: RESEARCH PAPER}

Keywords:

Citation: Silviu Ursache, Eduard Lunca, Alexandru Salceanu, lonel Pavel, Analysis of the influence of the current drawn by the appliance on the close magnetic field, Acta IMEKO, vol. 7, no. 4, article 12, December 2018, identifier: IMEKO-ACTA-07 (2018)-04-12

Editor: Vilmos Pálfi, Budapest University of Technology and Economics, Hungary

Received April 24, 2018; In final form October 2, 2018; Published December 2018

Copyright: (C) 2018 IMEKO. This is an open-access article distributed under the terms of the Creative Commons Attribution 3.0 License, which permits unrestricted use, distribution, and reproduction in any medium, provided the original author and source are credited

Corresponding author: Silviu Ursache, e-mail: silviu_ursache@tuiasi.ro

\section{INTRODUCTION}

The rapid development of technology has been materialized over time by, for example, the creation and widespread use of household appliances.

At home or in the office, we are surrounded by the most sophisticated equipment and devices, without which it would be impossible for us to imagine our everyday living. Once connected to a power supply network, these devices generate low-frequency electric and magnetic fields, complementary to (operational) radio frequency fields. There is a qualitative perception that as power consumption increases, the generated (electro)-magnetic fields become more profound.

Concerning control of a person's exposure to electromagnetic fields during working hours, Directive 2013/35/EU [1] stipulates precise responsibilities for employers and presents an overview of action levels at $50 \mathrm{~Hz}$ $(1,000 \mu \mathrm{T}$ RMS $)$ as recommended by the ICNIRP 2010 Guidelines. At home, where a person is supposed to be in a "corner" for rest and relaxation, it is a priori supposed that exposure to various harmful factors, including electromagnetic fields, is lower, without additional checks. Is this true, given that the number and diversity of equipment found in a modern home can in some cases be much higher than at work? Sometimes, they are much closer to two of the most sensitive parts of the human body: the eyes and the brain. Most of the research undertaken on this issue has been focused on the work environment, with much less work dedicated to the private home environment. The variety of these low-frequency magnetic field sources involves a wide range of measurement methods, with the aim of realistically characterizing the electromagnetic home environment.

Even if numerous studies have been published over the last 25 years related to human exposure to low-frequency electric and magnetic fields [2-6], the possible connection between the magnetic field generated by appliances and the current consumption has not been investigated in detail.

More recent studies have started to approach this issue, making the necessary difference between occupational and residential exposure $[7,8]$.

Several scientific papers published in the last five years have taken into account the classification of household appliances operation cycles, which is beneficial to the Smart Grid concept 
[9], or classification using probabilistic neural networks [10]. Systems monitoring electrical energy consumption have also been developed, allowing for automatic home appliance detection (and even identification) by means of the analysis of domestic power consumption [11-13].

Recent papers have presented techniques and various algorithms for monitoring electric power consumption in individual households $[14,15]$.

An important concern of European organizations and authorities is the characterization and limitation of household electricity consumption, promoting energy-saving techniques by means of adequate policies and recommendations [16].

In the relevant literature, the two most important factors that contribute to the characterization of a household electrical appliance, the absorbed current and the magnetic field emitted during standard operation, have been treated as separate. What we attempt in this paper is to study the possible relationship between the two previously mentioned characteristics.

In particular, we select 47 representative household appliances, mainly because they work in very close vicinity to the human body. We establish their normal, stable operating regime and measure both the current absorbed from the outlet and the resulting magnetic field, at a point located $30 \mathrm{~cm}$ from the apparatus. We chose this distance as a reasonable compromise for the cases in which the user stands close to the equipment for comparative consistency. Consequently, the test setup consists of an adequate current sensor and one magnetic flux density measuring equipment. Several factors are taken into account: the specifications of the measurement devices, the measurement methods, and the operating mode of the appliances.

\section{METHODOLOGY AND INSTRUMENTATION}

All magnetic field measurements were performed using a hand-held triple axis 480826 EMF Tester manufactured by Extech Instruments (Figure 1), which operates in the frequency range from $30 \mathrm{~Hz}$ to $300 \mathrm{~Hz}$, the RMS values of the magnetic flux density being calculated by Equation (1):

$$
B=\sqrt{B_{x}^{2}+B_{y}^{2}+B_{z}^{2}},
$$

where $B_{x}, B_{y}$, and $B_{z}$ are the RMS values of the magnetic flux density measured in three orthogonal directions.

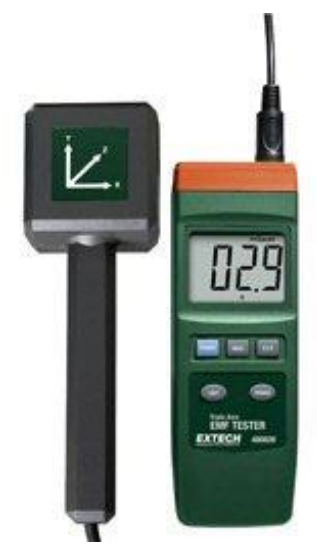

Figure 1. A triple-axis 480826 EMF tester manufactured by Extech Instruments.

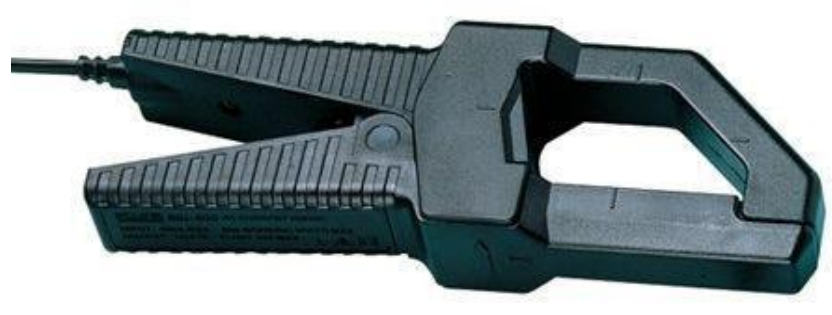

Figure 2. 80i-400 AC true RMS current clamp meter manufactured by Fluke.

The intensity of the drawn current was measured using a 80i400 AC true RMS current clamp meter produced by Fluke, with the output level set at $1 \mathrm{~mA}$ per $1 \mathrm{~A}$ of input current (Figure 2).

The measuring setup for studying the possible relationship between the magnetic field generated by the home appliance (Equipment Under Test [EUT]) and current consumption is shown in Figure 3.

Both in terms of the average and RMS values of the absorbed current, from the point of view of the density of the produced magnetic flux and of the desired modeling simulation, the household appliances can be divided into two main categories (that may also have common elements): (quasi-) linear devices based on the thermal effect of electric energy and appliances with a motor that converts electrical energy into mechanical energy. There are, of course, appliances that meet both criteria of having both heating resistance and an electric motor (washing machines, air-conditioning devices, microwave ovens, etc.).

Consequently, the 47 studied appliances have been categorized as either Class 1 (C1) appliances that mainly operate based on the thermal effect of the electric current and Class 2 (C2) appliances that essentially operate based on an electric motor(s), with more details in [17].

For both categories, the real, direct measurements have been performed with the handheld triple-axis 480826 EMF Tester.

For the purpose of comparison, the magnetic field generated by a linear conductor or an operating electric motor could be calculated using various commercial software packahes, mostly based on the finite element method.

We have also performed the theoretical calculation of the magnetic flux density generated by C1-type appliances based on their drawn current by using the Biot-Savart law. This calculation provides that the derivative of the magnetic flux density $d \vec{B}$ is generated at a point $M$, at distance $r$ (direction is given by the unit vector $\hat{r}$ ) from the current $I$ (which crosses the infinitesimal

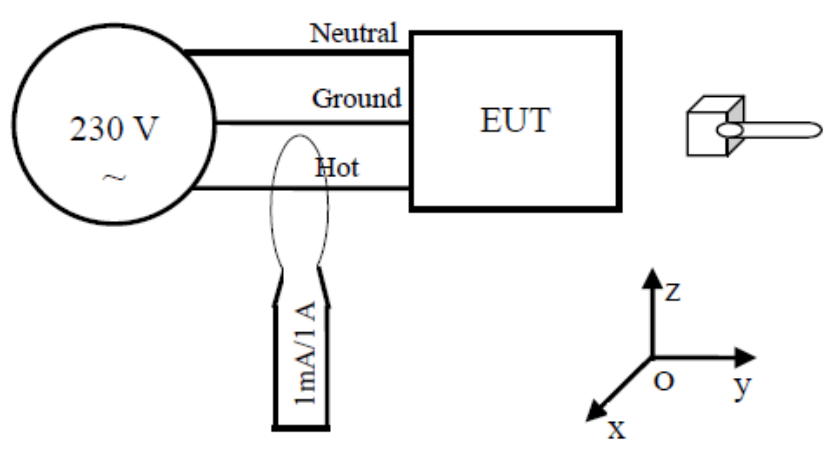

Figure 3. Experimental setup. 
length), with the current carrying wire $d \vec{l}$, as shown in Equation (2):

$$
d \vec{B}=\frac{\mu_{0} I}{4 \pi} \frac{d \vec{l} \times \hat{r}}{r^{2}}
$$

The relationship between the drawn current and the magnetic field is studied considering a long straight wire, which is carried out by an electric current $I$ in the positive direction along the $y$ axis.

We essentially want to calculate the magnetic field $\vec{B}$, generated at perpendicular distance $\mathrm{R}$ from the wire due to the current running in the positive direction along the $y$-axis.

We have chosen the origin $O$ as being the separation between the negative and positive parts of the wire related to the $x$-axis. The wire is divided into an infinite number of small segments $d$, each of which is driven by an infinitesimal current, known as the "current element". This current element creates an infinitely small magnetic field, given by $d B$ at point $M$. If we want to find the total magnetic flux density at this point, we add all the quantities of $d \vec{B}$ as a result of all the small current elements given by $d l$, as shown in Equation (3):

$$
\vec{B}=\sum d \vec{B}
$$

Because we have considered an infinitely long wire, Equation (3) can be written in integral form, as shown in Equation (4):

$$
\vec{B}=\frac{\mu_{0} I}{4 \pi} \int_{-\infty}^{+\infty} \frac{d \vec{l} \times \hat{r}}{r^{2}}=\frac{\mu_{0} I}{4 \pi} \int_{-\infty}^{+\infty} \frac{d \vec{l} \sin \varphi}{r^{2}}
$$

where $\varphi$ is the angle between $d \vec{l}$ and $\vec{r}$.

We simply have to use the right triangle from Figure 4, where $\mathrm{R}$ is the distance from point $M$ to the origin point $O$, and $l$ is the distance from origin $O$ to the $d l$ quantity.

By using the Pythagorean Theorem, we have:

$$
r^{2}=R^{2}+l^{2}
$$

where $R$ is considered constant, while $l$ and $\varphi$ are variables.

Both $l$ and $\varphi$ are variables appearing in Equation (4), and it is necessary to express one in terms of the other.

We want to express $l$ in terms of $\varphi$ using the tangent function, as shown in Equation (6):

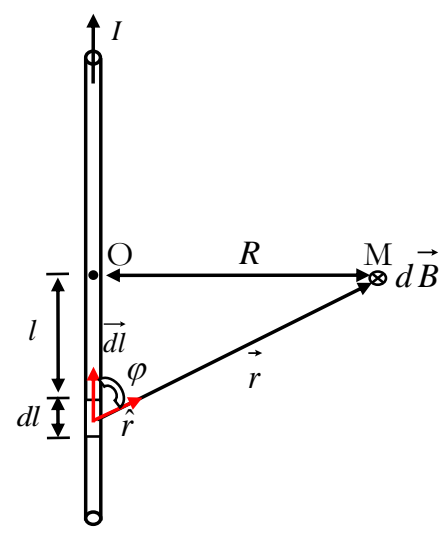

Figure 4. Applying Biot-Savart law for calculating the magnetic flux density.

$$
\tan \varphi=-\frac{R}{l}
$$

We observe that:

$$
l=-\frac{R}{\tan \varphi}
$$

We also want to express $d y$ in terms of angle $\varphi$, deriving both members of Equation (7) as presented in Equation (8):

$$
d l=\frac{R d \varphi}{\sin ^{2} \varphi}=\frac{R d \varphi}{\left(\frac{R}{r}\right)^{2}}=\frac{r^{2} d \varphi}{R} .
$$

Using (8) in (4), we get the equation (9):

$$
|\vec{B}|=\frac{\mu_{0} I}{4 \pi} \int_{0}^{\pi} \frac{r^{2} d \varphi \sin \varphi}{r^{2} R}=\frac{\mu_{0} I}{4 \pi R} \int_{0}^{\pi} d \varphi \sin \varphi
$$

In Equation (9), we observe that the integral is defined from 0 to $\pi$. In Figure 4, we observe that when the negative part of the wire goes to $-\infty$, the angle $\varphi$ becomes very small, in fact, very close to zero. While the wire tends to $+\infty$, the angle goes to $\pi$ radians.

After some related transformations, we get Equation (10):

$$
|\vec{B}|=\frac{\mu_{0} I}{4 \pi R} \int_{0}^{\pi} d \varphi \sin \varphi=-\left.\frac{\mu_{0} I \cos \varphi}{4 \pi R}\right|_{0} ^{\pi}=\frac{\mu_{0} I}{2 \pi R} .
$$

In essence, the Biot-Savart law establishes the direct proportionality between the electric current and the generated magnetic field.

\section{MEASUREMENTS AND RESULTS}

\subsection{Household appliances based on the electro-thermal effect}

Using the setup in Figure 3, we have determined the absorbed current and the magnetic field emitted by 21 devices. The registered data is summarized in Table 1. For comparison, the last column on the right, $\mathrm{Bc}$, is introduced. This column contains the magnetic field values, calculated on the basis of the BiotSavart law, for the measured currents, at the points with the previously stated spatial coordinates. The household appliances have been modeled as a long straight wire through which a known electric current passes.

The values recorded for the drawn current range from $1.5 \mathrm{~A}$ (a washing machine in extortion mode) to $22.3 \mathrm{~A}$ (an electric hob).

For comparable (even quasi-similar) appliances, it may be that both the drawn current and the magnetic field differ significantly. Furthermore, the direct proportional dependence that theoretically might be established between the current and the field, according to the Biot-Savart law, is by no means respected. For instance, the two irons, labelled "iron3" and "iron 4" have quasi-similar values for the drawn current (about $8.6 \mathrm{~A}$ ), while associated magnetic field values differ with an order of magnitude (628.89 and 52.81, respectively).

The fact that there are significant differences between the magnetic fields emitted by devices with perfectly comparable features is evidence that this issue has been considered by many manufacturers and designers, who have tried to find the right technological solutions (shielding, wiring, grounding, positioning, etc.) with the aim of reducing the (electro)magnetic emissions.

\subsection{Household appliances based on an electric motor}


Table 1. Class C1 devices: typical values of magnetic flux density and drawn current at $30 \mathrm{~cm}$ distance.

\begin{tabular}{|c|c|c|c|}
\hline Household appliance & $I$ in $A$ & $B$ in $n T$ & $B_{c}$ in $n T$ \\
\hline Kettle Mug 1 & 8 & 4696.8 & 5333.33 \\
\hline Kettle Mug 2 & 8.6 & 5517.25 & 5733.33 \\
\hline Sandwich-maker & 2.85 & 144.57 & 1900 \\
\hline Toaster 1 & 5.2 & 361.53 & 3466.67 \\
\hline Toaster 2 & 3.37 & 259.62 & 2246.67 \\
\hline Coffee-maker & 4.1 & 67.82 & 2733.33 \\
\hline Electric boiler & 5.1 & 708.59 & 3400 \\
\hline Hairdryer 1 & 3.26 & 42.43 & 2173.33 \\
\hline Hairdryer 2 & 2.16 & 274.40 & 1440 \\
\hline Hairdryer 3 & 3.94 & 746.79 & 2626.67 \\
\hline Hairdryer 4 & 6.3 & 1723.36 & 4200 \\
\hline Electric hob & 22.3 & 87.74 & 14866.67 \\
\hline Electric heating & 6.25 & 64.03 & 4166.67 \\
\hline Iron 1 & 5.02 & 120.83 & 3346.67 \\
\hline Iron 2 & 6.3 & 2800 & 4200 \\
\hline Iron 3 & 8.65 & 628.89 & 5766.67 \\
\hline Iron 4 & 8.62 & 52.81 & 5746.67 \\
\hline Electric tap & 24.1 & 946.04 & 16066.67 \\
\hline Electric oven & 8.22 & 522.78 & 5480 \\
\hline Washing machine extortion & 1.5 & 960.10 & 1000 \\
\hline Washing machine lavation & 9 & 204.21 & 6000 \\
\hline
\end{tabular}

Table 2. Typical values of magnetic flux density and drawn current at $30 \mathrm{~cm}$ for C2 class appliances.

\begin{tabular}{|c|c|c|}
\hline Household appliance & $I$ in $A$ & $\mathbf{B}$ in $\mathbf{n T}$ \\
\hline Vegetable chopper 1 & 0.1 & 207.12 \\
\hline Mixer & 0.29 & 142.48 \\
\hline Hand blender & 0.26 & 580.68 \\
\hline Electric oven & 8.34 & 383.27 \\
\hline Freezer 1 & 6.4 & 158.42 \\
\hline Freezer 2 & 1 & 136.38 \\
\hline Freezer 3 & 1.8 & 400.50 \\
\hline Refrigerated display case 1 & 0.21 & 238.74 \\
\hline Refrigerated display case 2 & 0.26 & 81.24 \\
\hline Vertical freezer & 1.02 & 74.83 \\
\hline Microwave oven 1 & 5.75 & 5101.96 \\
\hline Microwave oven 2 & 4.9 & 9053.86 \\
\hline Microwave oven 3 & 2.9 & 10080 \\
\hline Microwave oven 4 & 5.88 & 3379.18 \\
\hline Refrigerator 1 & 0.3 & 17.32 \\
\hline Refrigerator 2 & 0.34 & 99.50 \\
\hline Refrigerator 3 & 0.73 & 64.03 \\
\hline Refrigerator 4 & 0.4 & 75.49 \\
\hline Hand-mixer & 0.45 & 9129.75 \\
\hline Vacuum cleaner 1 & 6.15 & 756.96 \\
\hline Vacuum cleaner 2 & 3.05 & 780.12 \\
\hline Coffee grinder & 0.4 & 433.7 \\
\hline Fruit juicer & 0.13 & 78.74 \\
\hline Massage machine & 0.05 & 264.76 \\
\hline Meat machine & 0.22 & 677.86 \\
\hline Cooker hood & 0.75 & 354.96 \\
\hline
\end{tabular}

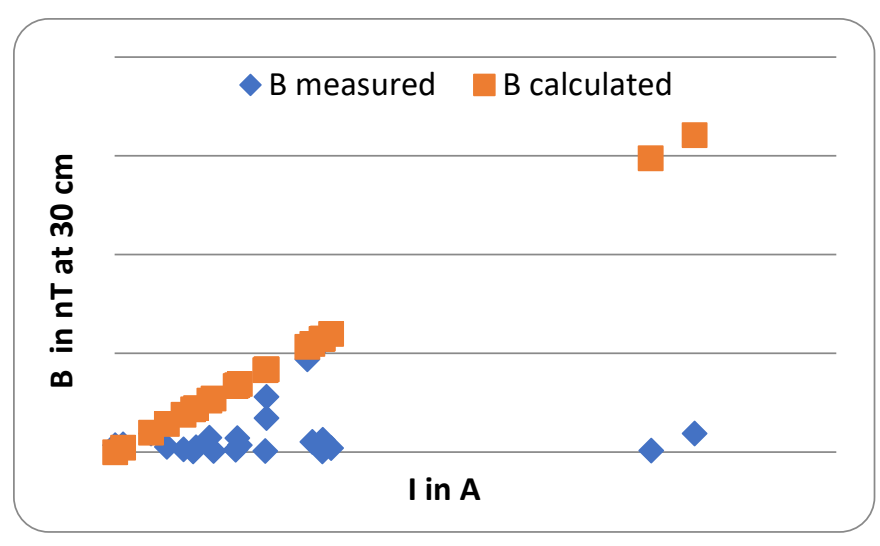

Figure 5. The dependence between the magnetic field (measured vs. calculated) and drawn current for $\mathrm{C} 1$ Class appliances

We performed measurements on the drawn current and the emitted magnetic field for 26 household appliances, Class C2, summarized in Table 2.

Most of the recorded values for the drawn current are smaller than $1 \mathrm{~A}$, excluding microwave ovens, electric vegetable cutters, and vacuum cleaners, for which the current consumption ranges from $2.9 \mathrm{~A}$ to $8.34 \mathrm{~A}$.

Perhaps the results regarding the two vacuum cleaners belonging to the same class should be considered. Although the absorbed currents are in the 1:2 ratio, the magnetic fields emitted are virtually equal.

We also remarked that in the case of a hand-mixer, the drawn current has a very low value, while the magnetic flux density is greater than $9 \mu \mathrm{T}$.

In the case of several household appliances, such as refrigerators, fruit juicers, or vertical freezers, very low values for both the drawn current and magnetic flux density have been recorded.

As we anticipated, there is no linear dependence between the current consumption and the magnetic field generated by the household appliances from Class C2. Figure 6 graphically expresses this result.

It is appropriate to suggest the absence of any law of dependence between the current absorbed by the appliance and its emitted magnetic field by combining the graphical representations of Figure 5 and Figure 6, thus resulting in Figure 7.

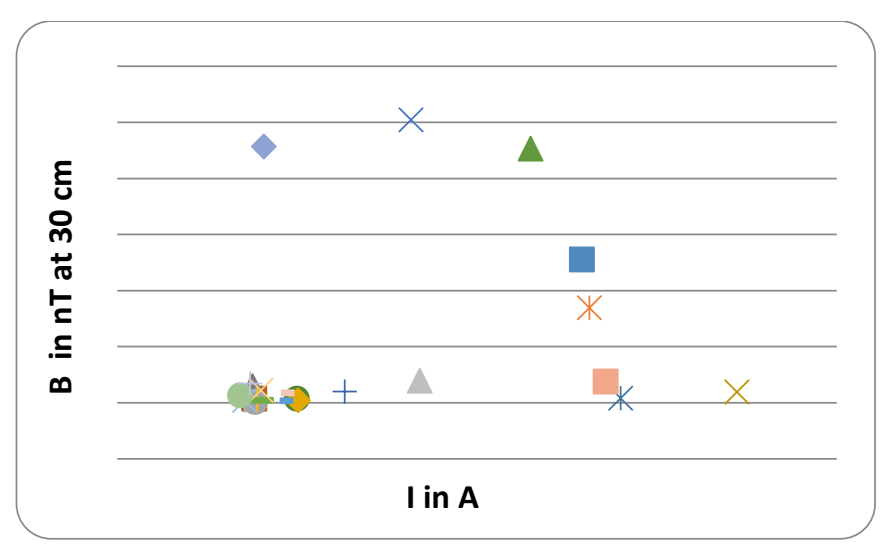

Figure 6. Correspondence between the magnetic field and drawn current for Class C2 appliances. 


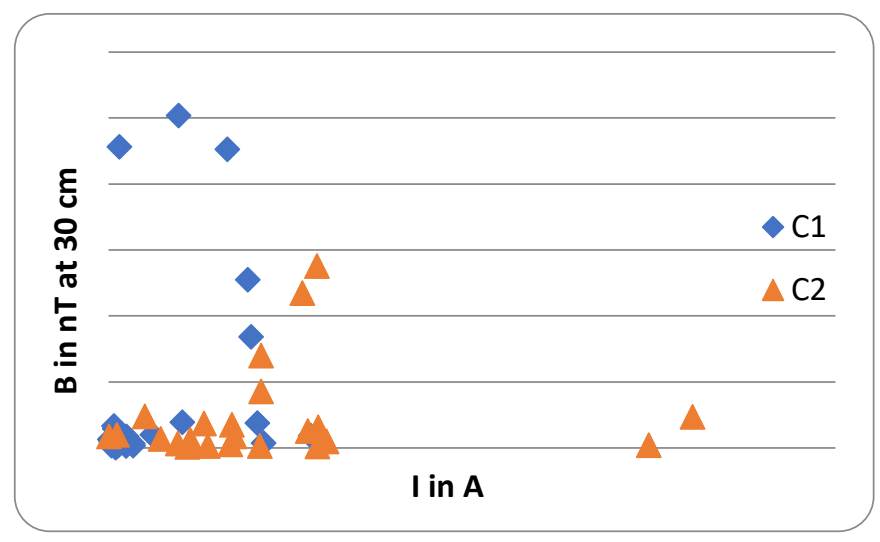

Figure 7. The dependence between the magnetic field and drawn current both for $\mathrm{C} 1$ and $\mathrm{C} 2$ Class appliances.

\section{CONCLUSIONS}

By means of a simplistic application of the well-known BiotSavart law, at first glance, a user with good knowledge of electrical engineering would be tempted to consider that a highpower appliance (which consumes a higher current from the network) would produce a high magnetic field in its vicinity. Rigorous measurements made for 47 household appliances have shown that the situation is different, primarily due to designers who have adopted technological solutions (shielding, wiring, cabling, grounding, positioning, etc.) to reduce or simply compensate for low-frequency magnetic field pollution. It should also be borne in mind that the distribution of the magnetic field around an electric motor rarely has spatial uniformity. Thus, the (horizontal) position of the measuring point, located on a $30 \mathrm{~cm}$ radius circumference, might influence the measured value.

In future research, we aim to 3D model the spatial distribution of magnetic fields produced by electric motors that typically equip household appliances. Furthermore, a comparative analysis of the efficiency of the methods adopted by various designers regarding the diminution of low-frequency magnetic fields will be performed.

\section{References}

[1] A. Salceanu, E. Lunca, M. Paulet, Affordable evaluation of lowfrequency electric fields from the standpoint of Directive 2013/35/EU, ACTA IMEKO 6(4) (2017) pp. 37-45.

[2] E. Lunca, S. Ursache, A. Salceanu, Study of the power-frequency magnetic fields in residences and schools, Bulletin AGIR 3 (2012) pp. 689-694.

[3] E. Ali, A. R. Memari, "Effects of magnetic field of power lines and household appliances on human and animals and its mitigation", Proc. of the IEEE Middle East Conference on Antennas and Propagation, 20-22 Oct., 2010, Cairo, Egypt, DOI: 10.1109/MECAP.2010.5724167

[4] W. T. Kaune, Assessing human exposure to power-frequency electric and magnetic fields, Environmental Health Perspectives 101 (1993) pp. 121-133.
[5] A. Cieśla, W. Kraszewski, M. Skowron, P. Syrek, Determination of safety zones in the context of the magnetic field impact on the surrounding during magnetic therapy, Przegląd Elektrotechniczny 87(7) (2011) pp. 79-82.

[6] P. Syrek, M. Skowron, "The impact of overhead lines for employees with stents", Proc. of the IOP Conference Series: Materials Science and Engineering, 2017, 200(012013), pp. 1-6.

[7] S. Ursache, A. Salceanu, O. Neacsu, "Indoor and outdoor measurements of the low frequency magnetic fields in an urban area", Proc. of the International Conference and Exposition on Electrical and Power Engineering (EPE), 2016, pp. 1-4.

[8] M. Chen, Y. Du, "Magnetic field environments at power frequency inside modern buildings", Proc. of the Asia-Pacific Symposium on Electromagnetic Compatibility (APEMC), 2013, DOI: $10.1109 /$ APEMC.2013.7360626.

[9] Z. Wang, R. Srinivasan, Classification of Household Appliance Operation Cycles: A Case-Study Approach, Energies 8 (2015), pp. 10522-10536.

[10] N. A. Rosdi, F. H. Nordin, A. K. Ramasamy, N. B. Mustafa, "Classification of electrical appliances using magnetic field and probabilistic neural network", Proc. of the $5^{\text {th }}$ IEEE Control and System Graduate Research Colloquium, 2014, DOI: 10.1109/ICSGRC.2014.6908735.

[11] R. Lukaszewski, K. Liszewski, W. Winiecki, "Methods of electrical appliances identification in systems monitoring electrical energy consumption", Proc. of the $7^{\text {th }}$ IEEE International Conference on Intelligent Data Acquisition and Advanced Computing Systems (IDAACS), DOI: 10.1109/IDAACS.2013.6662630.

[12] R. Streubel, B. Yang, "Identification of electrical appliances via analysis of power consumption", Proc. of the Third International Conference on Computer, Communication, Control, and Information Technology (C3IT), 2015, DOI: 10.1109/C3IT.2015.7060219.

[13] H. Serra, J. Correia, António J. Gano, António M. de Campos, Isabel Teixeira, "Domestic power consumption measurement and automatic home appliance detection", Proc. of the IEEE International Workshop on Intelligent Signal Processing, 2005, DOI: 10.1109/WISP.2005.1531645.

[14] M. Fitta, S. Bizaet, M. Lehtonen, G. Jacucci, Exploring techniques for monitoring electric power consumption in households", Proc. of UBICOMM 2010: The $4^{\text {th }}$ International Conference on Mobile Ubiquitous Computing, Systems, Services, and Technologies, 2010, pp. 471-477.

[15] W. J. Jin, Y. Feng, N. Zhou, X. Zhong, "A household electricity consumption algorithm with upper limit", Proc. of the International Conference on Wireless Communication and Sensor Network, 2014, DOI: 10.1109/WCSN.2014.93.

[16] A. Almeida, P. Fonseca, Barbara Schlomann, Nicolai Feilberg, Characterization of the household electricity consumption in the EU, potential energy savings, and specific policy recommendations, Energy and Buildings 43(8) (2011) pp. 18841894

[17] S. Ursache, E. Lunca, A. Salceanu, I. Pavel, "Study on the relationship between magnetic fields generated by home appliances and associated drawn currents", Proc. of the $22^{\text {nd }}$ IMEKO TC4 International Symposium and 20 ${ }^{\text {th }}$ International Workshop on ADC Modelling and Testing, Sept. 14-16, 2017, Iasi, Romania, pp. 305-308. 